\title{
Polarisation Dependent Dynamics in Bulk Semiconductor Optical Amplifiers
}

\author{
S. Philippe ${ }^{1 *}$, A.L. Bradley ${ }^{1}$, F. Surre ${ }^{2}$, P. Landais ${ }^{2}$, Member, IEEE, B. Kennedy ${ }^{3}$, M. Martinez-Rosas ${ }^{4}$ \\ ${ }^{I}$ School of Physics, Trinity College Dublin, Dublin, Ireland \\ ${ }^{2}$ School of Electronic Engineering, Dublin City University, Dublin, Ireland \\ ${ }^{3}$ Departamento de Ingenieria Electrica, Universidad de Santiago, Chile \\ ${ }^{4}$ Universidad Autonoma de Baja California, Ensenada, Mexico \\ *Tel: +35316082677,Fax: +35316711759,Email:philipps@tcd.ie
}

\begin{abstract}
A free space contra-propagation set-up is implemented and a dynamic pump-probe study of the eigenmodes of a SOA in the picosecond regime is undertaken. The time-resolved probe transmission of the TE and TM modes of the device is measured for the co and cross-polarised cases as a function of pump pulse energy. The relative contributions of interband and intraband processes to the gain compression are studied, as well as the coupling between the TE and TM modes. The high gain compression due to intraband effects observed in the TETE case is of particular interest for ultrafast all-optical switching.
\end{abstract}

Keywords: semiconductor optical amplifier, gain, polarisation, pump-probe, optical switching.

\section{INTRODUCTION}

Semiconductor optical amplifiers (SOAs) exhibit non-linearities that make them suitable for high-speed signal processing applications. They are polarisation sensitive, with different gain and refractive indices in the TE and TM modes [1]. Depending on the chosen configuration, polarisation can either be taken advantage of, using cross-gain and cross-phase modulation for all-optical wavelength conversion [2-5] or have to be minimized. They are however always present and should be well understood in order to optimize any SOA-based all-optical wavelength conversion scheme. A number of applications based on non-linear polarisation rotation have been presented, however fiberised set-ups are commonly used, making it difficult to set and maintain the state of polarisation of the signals.

Conventional pump-probe experiments use a co-propagation configuration, where the pump and probe signals travels collinearly through the SOA and are separated at the output using a polarizer. In that case the pump and probe output states of polarisation have to be linear and orthogonal to each other, practically restricting the input states of polarisation to the eigenmodes of the device, pump TE probe TM and pump TM probe TE. No restrictions on the signals polarisation apply when a contra-propagation configuration is used and the probe and pump signal are separated by a beamsplitter. We propose to use a free space contra-propagation configuration and present a polarisation dependent pump-probe study of a bulk SOA in the picosecond regime. In this paper we focus on the behaviour of the SOA when light is injected along the eigenmodes of the device, with comparison of co and cross-polarised cases.

\section{EXPERIMENT}

\subsection{Experimental set-up}

A free space contra-propagation configuration is used, allowing total control and preservation of the state of polarisation of the injected and collected signals, as shown on Fig. 1. The $1580 \mathrm{~nm}$ pulsed input is produced using a fibre based femtosecond laser, with a repetition rate of $82 \mathrm{MHz}$. The pulse width after dispersion in the fiberised output is $2.5 \mathrm{ps}$ and a grating filter with a $1 \mathrm{~nm}$ spectral width is used. The pulses are coherent and one is delayed with respect to the other using a variable delay stage. The polarisation of each beam is controlled independently with a quarter wave plate (QWP) and a half wave plate (HWP). Two beamsplitters allow us to monitor the input and output of each beam. The light is coupled in and out of the SOA by two anti reflectioncoated aspheric lenses mounted on micro-control 3D translation stages. The device under test is a commercially available $1.5 \mathrm{~mm}$ long bulk InGaAsP/InP travelling wave SOA biased at $350 \mathrm{~mA}$ and temperature regulated at $20^{\circ} \mathrm{C}$ by means of a Peltier cooler. An interference filter is used to isolate the probe signal from the broad ASE at $1580 \mathrm{~nm}$ and the signal is detected using a lock-in technique. 


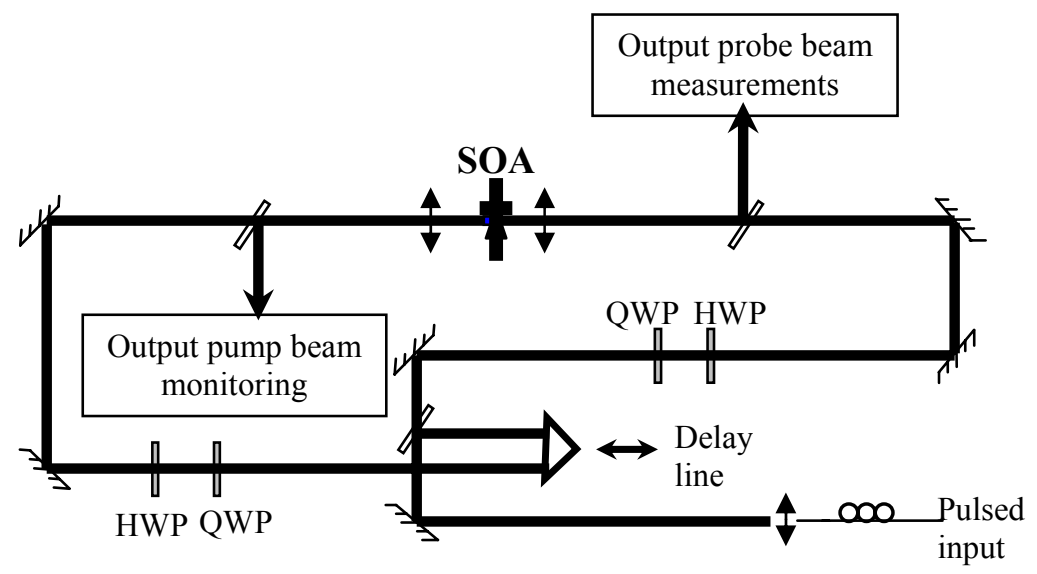

Figure 1. Experimental set-up.

\subsection{Contra-propagation}

As most of previous pump-probe studies of SOAs have been performed in a co-propagation configuration, it is useful to stress the main differences between co and counter propagation. Indeed the transmission curves can look rather similar while the process is different. Unlike co-propagation where there is only one value of the delay for which the pulses overlap, in counter-propagation for a range of time delays, the pump and probe pulses overlap at different positions inside the SOA. As a consequence, in this geometry it is important to note that the displacement of the delay line between the pulses overlapping at the input and them overlapping at output corresponds to twice the length of the SOA.

Probe signals obtained in counter-propagation are shown in Fig. 2. We refer to the probe input facet (pump output facet) as the SOA input and the probe output facet (pump input facet) as the SOA output. Before A, at more negative delays, the probe leaves the SOA before the pump enters it and its transmission is left unchanged. At A, the pump and probe pulses overlap at the output of the SOA. Between A and B the pulses overlap inside the SOA, and as is expected the gain experienced by the probe will be reduced and therefore the intensity of the amplified probe signal will decrease. A minimum is reached at $\mathrm{B}$, where the pulses overlap at the input of the SOA. This minimum can be expected, as it is at this delay that the pump has affected the entire length of the SOA, with no time for any recovery before the probe arrives. After that point the pump leaves the SOA before the probe enters it and the device has started to recover before the arrival of the probe. As the delay is further increased the amplified probe signal will continue to increase and the recovery time of the device is monitored. The recovery shows two components: a faster recovery component between $\mathrm{B}$ and $\mathrm{C}$, followed by slower recovery due to carrier density pulsation occurring on the timescale of the order of hundreds of picoseconds.

\section{RESULTS AND DISCUSSION}

The pump and probe wavelength are set at $1580 \mathrm{~nm}$ at the peak of the SOA gain spectrum. The probe transmission is recorded as a function of pump probe delay for a range of pump pulse energies $(6 \mathrm{fJ}-975 \mathrm{fJ})$, while the probe pulse energy is kept constant at $12 \mathrm{fJ}$, below the gain saturation energy. The data are taken for the different combinations of probe and pump states of polarisation, probe and pump TE polarised (TETE), probe and pump TM polarised (TMTM), probe TE pump TM (TETM) and probe TM pump TE (TMTE).

The modification of the amplified probe signal as a function of delay and polarisation is shown on Fig. $2 a$ and $2 b$ for $24 \mathrm{fJ}$ and $732 \mathrm{fJ}$ pump energy respectively. The output intensity is normalised by the probe signal level that is achieved in the absence of the pump pulse. As expected there is onset of gain compression of the probe signal at delays where the pump pulse has travelled through regions of the SOA in advance of the probe. As can be seen the response of the probe signal is dependent on the states of polarisation of the two signals and on pump pulse energy. Two components can be seen in the recovery of the probe transmission at positive delays. The fast recovery component is due to intraband effects such as carrier heating, spectral hole-burning, two photon absorption and free carrier absorption, while the slower recovery is associated with interband effects [6]. As the pump pulse duration decreases the contribution of intraband effects to the gain compression increases. Even though the timescales for the intraband effects (typically $<500 \mathrm{fs}$ ) cannot be resolved within the time resolution of our $2.5 \mathrm{ps}$ pulses, we can determine the polarization dependence of the intra and interband dynamics by examining the relative contributions of the fast and slow components to the probe transmission as a function of delay. At low powers the fast recovery component is only evident in the co-polarised pump-probe configurations. The fast recovery component dominates the probe transmission recovery for the TETE case, indicating that intraband processes are most significant under these polarization conditions and only a small level of slow gain compression remains. The fast recovery component is also evident for the TMTM case, but the slow gain compression (measured between $\mathrm{A}$ and $\mathrm{C}$ ), shown on Fig. $2 a$, is larger than for TETE, indicating a greater 
contribution of interband effects. As the pump power is increased the interband effects increasingly dominate the gain compression for TMTM and are larger than from TETE at all pump energies, see Fig. $3 a$. Similarly the fact that there is no fast component in the cross-polarised cases at low power indicates the gain compression of the probe signal is a result of interband dynamics under those conditions. Intraband effects are only evident in the cross-polarised cases at higher pump energies.
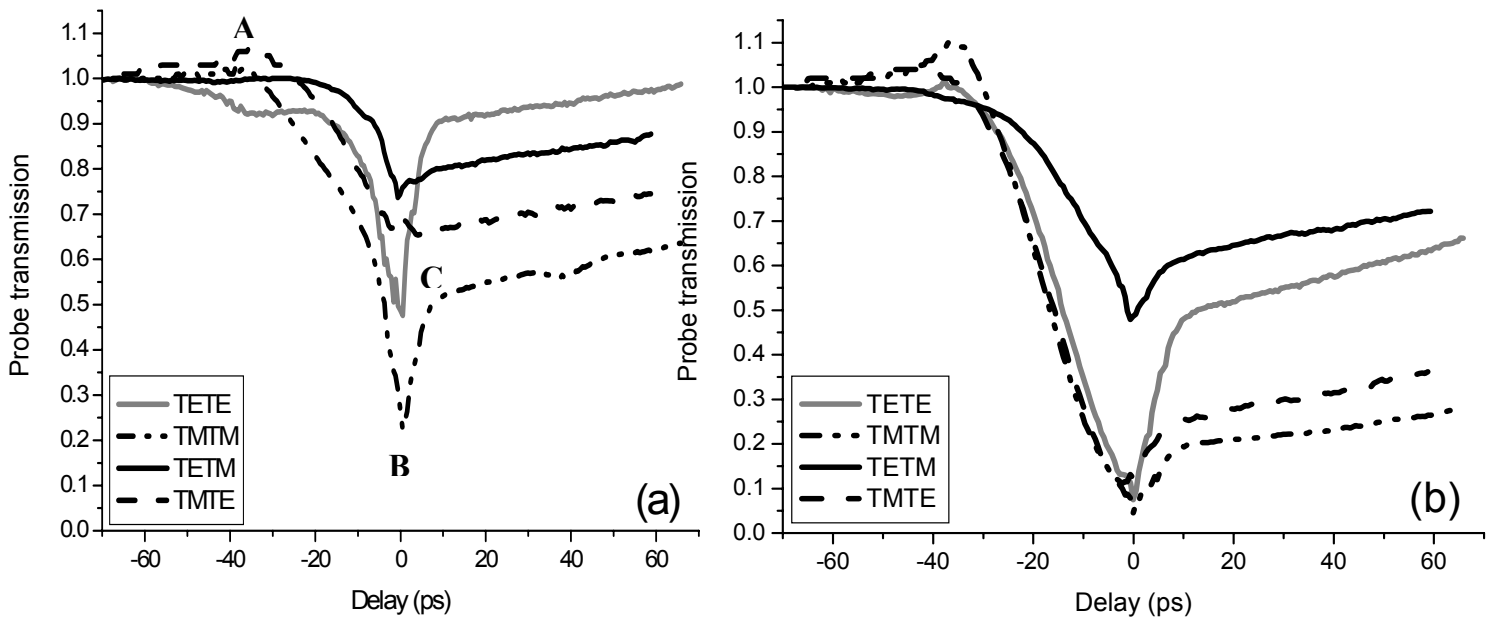

Figure 2. Transmitted pump signals as a function of delay for the different co and cross polarised cases:

$$
E_{\text {pump }}=24 \mathrm{fJ} \text {, (b) } E_{\text {pump }}=732 \mathrm{fJ} \text {. }
$$

At lower powers the onset of the decrease in the probe transmission occurs at earlier time delays for the copolarized pump and probe pulses due to the fact that the effect of the pump is greater and therefore it has to traverse less of the SOA to induce gain compression of the probe signal. An additional feature is observed around -30 ps delay when the pump and probe pulses overlap close to the output facet of the SOA. It can also be noted that this feature is strongly polarisation dependent. For high pump powers there is an increase in the probe transmission in the TMTM case, whereas the feature is absent in the TETM case at all pump energies. Modelling is under way to study the origin of this effect, which most likely arises from modification of the carrier distribution along the device [7].
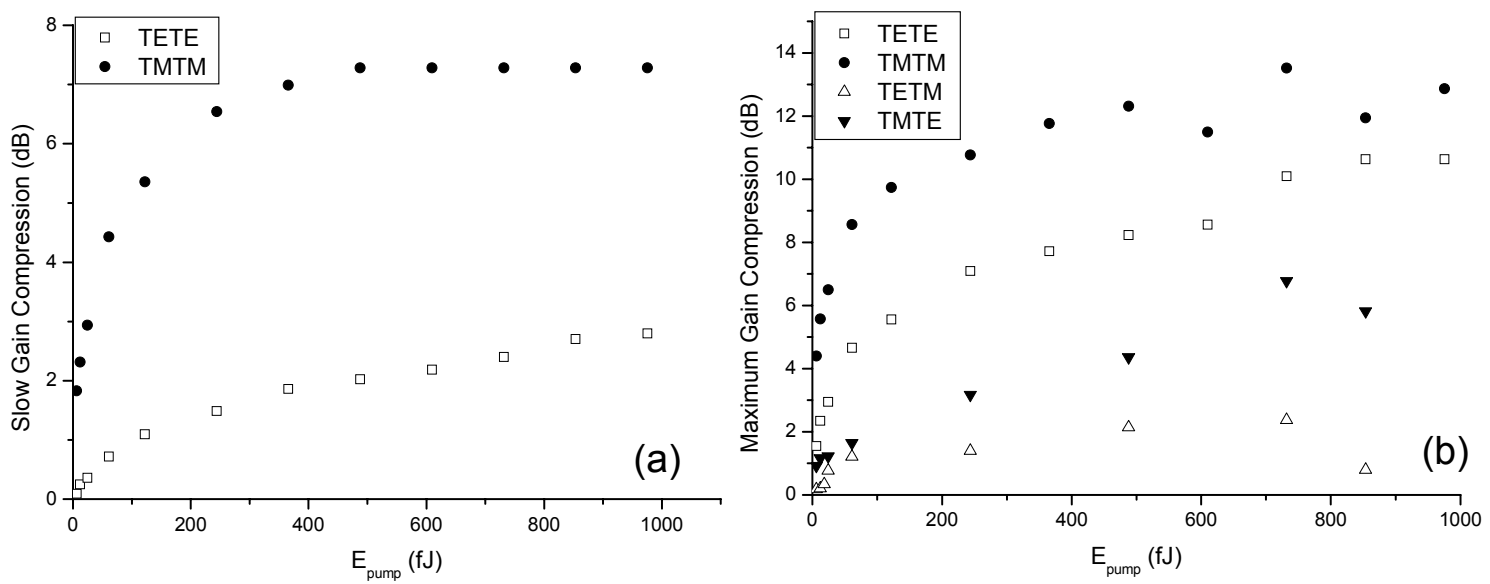

Figure 3. (a) slow gain compression, (b) maximum gain compression.

The maximum gain compression (measured between A and B) at zero time delay for each case is shown on Fig. $3 b$ as a function of time delay. As the pump energy increases the gain compression increases, as would be expected from carrier depletion effects. At higher pump energies the gain compression stabilises in the copolarised cases and starts to decrease in the cross-polarised cases. A higher gain compression is achieved by pumping and probing the same eigenmode. When pumping orthogonal to the probed mode, much higher pump energy is needed to reach the same gain compression. For the probe TM polarised, a gain compression of $\sim 7 \mathrm{~dB}$ can be achieved by either injecting a $24 \mathrm{fJ}$ TM polarised pump or a $732 \mathrm{fJ}$ TE polarised pump, giving a coupling coefficient of $3.3 \%$. Similarly, for the probe TE polarised, a gain compression of $\sim 2.5 \mathrm{~dB}$ is obtained with a $12 \mathrm{fJ}$ 
TE polarised pump or a 732 fJ TM polarised pump, giving a coupling coefficient of $1.6 \%$. Therefore coupling from one mode to the other is low but increases with pump energy and coupling from TE (pump) to TM (probe) is higher than TM to TE.

The discrepancy between the TM mode gain compression and the TE mode gain compression is most likely due to strain effects $[8,9]$. This is commonly present in bulk devices, even for a negligible lattice mismatch [8] and has the consequence of removing the degeneracy of the light and heavy hole bands. The carrier density of the light hole band is much lower than that of the heavy hole band. As the TM mode couples preferentially to the light holes [10], for the same pump input energy, we expect a higher gain compression in TM than in TE.

\section{CONCLUSION}

In this paper we have presented a series of polarization resolved pump probe experiments using a free space contra-propagation configuration, with co or cross polarized pulses. From the transmission of the probe pulse as a function of delay, it was possible to observe the fast and slow recovery components of the gain compression. The gain compression was found to be dependent on the pump and probe state of polarisation and pump pulse energy. The cross-polarised data are dominated by interband effects and show a low coupling between the modes. The maximum gain compression was found to be higher for the TMTM case but with a larger contribution of the slow gain compression than the TETE case. In conclusion the prevalence of the fast intraband effects in the TETE case shows the highest potential for ultrafast all-optical switching.

\section{REFERENCES}

[1] B. F. Kennedy, S. Philippe, P. Landais, A. L. Bradley, H. Soto, "Experimental investigation of polarisation rotation in semiconductor optical amplifiers," IEE Proceedings - Optoelectronics , vol. 151, no.2, pp. 114118, April 2004.

[2] M. Asghari, I. H. White, and R. V. Penty, "Wavelength conversion using semiconductor optical amplifiers," IEEE Journal of Lightwave Tech.,15, (7), pp.1181-1190, 1997.

[3] H. Soto, D. Erasme, and G. Guekos, "Cross polarization modulation in semiconductor optical amplifiers." IEEE Photon. Technol. Lett, 11, pp. 970-972, 1999.

[4] Y. Liu, M.T. Hill, E. Tangdiongga, H. de Waardt, N. Calabretta, G.D. Khoe, and H.J.S. Dorren, "Wavelength conversion using nonlinear polarization rotation in a single semiconductor optical amplifier," IEEE Photonics Tech. Letters, 15, (1), pp. 90-92, 2003.

[5] M.F.C.S tephens, M. Asghari, R.V. Penty, and I.H. White, "Demonstration of ultrafast all-optical wavelength conversion utilizing birefringence in semiconductor optical amplifiers," IEEE Photonics Tech. Letters, 9, (4), pp. 449-451, 1997.

[6] L. Occhi, Y. Ito, H. Kawaguchi, L. Schares, J. Eckner, G. Guekos, "Intraband Gain Dynamics in Bulk Semiconductor Optical Amplifiers: Measurements and Simulations," IEEE J. Quant. Elec., vol. 38, no. 1, January 2002.

[7] F. Ginovart, J. C. Simon, "Gain dynamics studies of a semiconductor optical amplifier," J. Opt. A: Pure Appl. Opt., 4, pp. 283-287, 2002.

[8] W. Wang, K. Alaart, D. Lenstra, "Gain Anisotropy in Semiconductor Optical Amplifier: Confinement Factors or Material Gain,” IEEE ICTON 2004, pp. 314-317.

[9] T. D. Visser, B. Demeulenaere, J. Haes, D. Lenstra, R. Baets, H. Blok, "Confinement and Modal Gain in Dielectric Waveguides," J. Lightwave. Tech., vol. 14, no. 5, May 1996.

[10] E. P. O'Reilly, A. R. Adams, "Band-Structure Engineering in Strained Semiconductor Lasers," IEEE J. Quant. Elec., vol. 30, no. 2, February 1994. 